\section{Primary Care Diagnosis of Gout Compared to a Primary Care Diagnostic Rule for Gout and to Classification Criteria}

\section{To the Editor:}

We read with interest the recent study by Dehlin and colleagues ${ }^{1}$, investigating the validity of a gout diagnosis in primary care. We have also investigated how a primary care diagnosis of gout compares to a primary care diagnostic rule for gout and to classification criteria. Our objective was to determine the proportion of patients with a primary care diagnosis of gout who fulfilled the primary care diagnostic rule by Janssens, et al for acute gouty arthritis ${ }^{2}$ and the 1977 American Rheumatism Association (ARA) criteria for the classification of acute arthritis of primary gout ${ }^{3}$.

Participants with gout undergoing followup as part of a prospective observational cohort study ${ }^{4}$ were sent a postal questionnaire, which included questions about clinical features of gout and comorbidities required to assess fulfillment of the Janssens diagnostic rule ${ }^{2}$ and the 1977 ARA criteria ${ }^{3}$. The cohort had been established in 2012 and recruited patients over the age of 18 years registered with 20 general practices in the West Midlands, UK. Participants were required to have a read-coded (diagnostic coding system used in UK general practice) consultation for gout or a prescription for allopurinol or colchicine within their medical records in the 2 years preceding baseline questionnaire mailing. Ethical approval was received from the North West - Liverpool East Research Ethics Committee (12/NW/0297). The highest serum urate level and the presence of tophi were extracted from medical records during the period from 2 years prior to baseline to 5 years post-baseline. Missing serum urate levels were assumed to be normal. A score of 8 or more for the Janssens diagnostic rule ${ }^{2}$ or 6 or more for the 1977 ARA criteria ${ }^{3}$ indicated a diagnosis of gout. The positive predictive value (PPV) for a primary care diagnosis of gout was calculated by dividing the number of participants who achieved the required score by the total number of participants.

The analysis included 536 participants; 484 (90.3\%) were male, with a mean (SD) age of 64.3 (11.3) years, and body mass index of 29.0 (5.4) $\mathrm{kg} / \mathrm{m}^{2}$. Median (interquartile range) gout duration was $10(3-22)$ years. Mean (SD) highest serum urate level was 370.2 (104.4) $\mu \mathrm{mol} / 1$, and $13(2.4 \%)$ participants had tophi recorded. The Janssens diagnostic rule and 1977 ARA criteria were fulfilled by 396 (PPV 73.9\%) and 429 (PPV 80.0\%) participants with a primary care diagnosis of gout, respectively. There were $464(86.6 \%)$ participants who fulfilled either the Janssens diagnostic rule or the 1977 ARA criteria.

The PPV of $73.9 \%$ for a primary care diagnosis compared to the Janssens diagnostic rule ${ }^{2}$ is similar to that reported by Dehlin and colleagues $(71 \%)^{1}$. When compared to the 1977 ARA criteria ${ }^{3}$, the PPV of $80 \%$ was similar to that reported by Janssens, et $a l^{5}$ in an earlier primary care study.

We also intended to calculate the PPV of a primary care gout diagnosis compared to the American College of Rheumatology/European League Against Rheumatism (ACR/EULAR) 2015 gout classification criteria ${ }^{6}$. However, 251 (46.8\%) of participants did not have a serum urate level recorded in their medical records. We were therefore unable to calculate a PPV compared to the ACR/EULAR 2015 criteria, because serum urate is a mandatory component of those criteria ${ }^{6}$. This suggests that the ACR/EULAR classification criteria may be difficult to apply retrospectively using routinely recorded data from primary care medical records. Because missing serum urate levels were assumed to be normal when deriving scores for the
Janssens diagnostic rule and 1977 ARA criteria, these estimates of PPV of a primary care diagnosis are likely to be conservative.

In this primary care cohort, the PPV of a primary gout diagnosis was high, with the majority of participants fulfilling the Janssens primary care diagnostic rule or 1977 ARA criteria. These findings provide reassurance about identifying research participants based on a clinical diagnosis in primary care where synovial fluid analysis and imaging are rarely performed.

\section{ACKNOWLEDGMENT}

This work received service support through the West Midlands North Clinical Research Network. The authors thank the administrative and health informatics staff at Keele University's Primary Care Centre Versus Arthritis, and staff and patients of the participating practices.

LORRAINE WATSON 10 , MSc, Research Fellow, Primary Care Centre Versus Arthritis, Research Institute for Primary Care and Health Sciences, Keele University; SARA MULLER ¿ , PhD, Senior Research Fellow, Primary Care Centre Versus Arthritis, Research Institute for Primary Care and Health Sciences; EDWARD RODDY ${ }^{\circ}$, DM, Reader in Rheumatology and Honorary Consultant Rheumatologist, Primary Care Centre Versus Arthritis, Research Institute for Primary Care and Health Sciences, Keele University, Keele, Staffordshire, UK, and Haywood Academic Rheumatology Centre, Midland Partnership National Health Service Foundation Trust, Haywood Hospital, Burslem, Staffordshire, UK. Address correspondence to L. Watson, Primary Care Centre Versus Arthritis, Research Institute for Primary Care and Health Sciences, Keele University, Staffordshire ST5 5BG, UK. E-mail: 1.watson@keele.ac.uk

\section{REFERENCES}

1. Dehlin M, Landgren AJ, Bergsten U, Jacobsson LTH. The validity of gout diagnosis in primary care - results from a patient survey. J Rheumatol 2019 Mar 22 [in press].

2. Janssens HJ, Fransen J, van de Lisdonk EH, van Riel PL, van Weel C, Janssen M. A diagnostic rule for acute gouty arthritis in primary care without joint fluid analysis. Arch Intern Med 2010;170:1120-6.

3. Wallace SL, Robinson H, Masi AT, Decker JL, McCarty DJ, Yü TF. Preliminary criteria for the classification of the acute arthritis of primary gout. Arthritis Rheum 1977;20:895-900

4. Chandratre P, Mallen C, Richardson J, Rome K, Bailey J, Gill R, et al. Prospective observational cohort study of Health Related Quality of Life (HRQOL), chronic foot problems and their determinants in gout: a research protocol. BMC Musculoskelet Disord 2012;13:1-7.

5. Janssens HJ, Janssen M, van de Lisdonk EH, Fransen J, van Riel PL, van Weel C. Limited validity of the American College of Rheumatology criteria for classifying patients with gout in primary care. Ann Rheum Dis 2010;69:1255-6.

6. Neogi T, Jansen TL, Dalbeth N, Fransen J, Schumacher HR, Berendsen D, et al. 2015 gout classification criteria: an American College of Rheumatology/European League Against Rheumatism collaborative initiative. Arthritis Rheumatol 2015;67:2557-68.

First Release July 15 2019; J Rheumatol 2019;46:11; doi: $10.3899 /$ jrheum.190495 